\title{
Controlling a mobile robot with a biological brain
}

Article

Published Version

Warwick, K., Xydas, D., Nasuto, S. J., Becerra, V. M., Hammond, M. W., Downes, J., Marshall, S. and Whalley, B. J. (2010) Controlling a mobile robot with a biological brain. Defence Science Journal, 60 (1). pp. 5-14. ISSN 0011-748X Available at https://centaur.reading.ac.uk/8181/

It is advisable to refer to the publisher's version if you intend to cite from the work. See Guidance on citing.

Published version at: http://publications.drdo.gov.in/ojs/index.php/dsj/article/view/11

Publisher: Defence Scientific Information \& Documentation Centre (DESIDOC), Delhi

All outputs in CentAUR are protected by Intellectual Property Rights law, including copyright law. Copyright and IPR is retained by the creators or other copyright holders. Terms and conditions for use of this material are defined in the End User Agreement.

\section{www.reading.ac.uk/centaur}

\section{CentAUR}

Central Archive at the University of Reading 
Reading's research outputs online 


\title{
Controlling a Mobile Robot with a Biological Brain
}

\author{
Kevin Warwick*, Dimitris Xydas*, Slawomir J. Nasuto*, Victor M. Becerra*, Mark W. Hammond*, \\ Julia H. Downes*, Simon Marshall ${ }^{* *}$ and Benjamin J. Whalley** \\ ${ }^{*}$ School of Systems Engineering, University of Reading, UK \\ E-mail:k.warwick@reading.ac.uk \\ ${ }^{* *}$ School of Chemistry, Food Biosciences and Pharmacy, University of Reading, UK
}

\begin{abstract}
The intelligent controlling mechanism of a typical mobile robot is usually a computer system. Some recent research is ongoing in which biological neurons are being cultured and trained to act as the brain of an interactive real world robot-thereby either completely replacing, or operating in a cooperative fashion with, a computer system. Studying such hybrid systems can provide distinct insights into the operation of biological neural structures, and therefore, such research has immediate medical implications as well as enormous potential in robotics. The main aim of the research is to assess the computational and learning capacity of dissociated cultured neuronal networks. A hybrid system incorporating closed-loop control of a mobile robot by a dissociated culture of neurons has been created. The system is flexible and allows for closed-loop operation, either with hardware robot or its software simulation. The paper provides an overview of the problem area, gives an idea of the breadth of present ongoing research, establises a new system architecture and, as an example, reports on the results of conducted experiments with real-life robots.
\end{abstract}

Keywords: Mobile robot, machine learning, neural network, biological neuronal networks, intelligent controlling mechanism, biological neurons

\section{INTRODUCTION}

The human brain is a complex computational platform with the ability to rapidly process vast amounts of information, adapt to noise, and tolerate faults. Recently, progress has been made towards hybrid systems which integrate biological neurons and electronic components. Reger ${ }^{1}$ demonstrated that it was possible to use the brain of a lamprey to control the trajectory of a robot whilst others were successfully able to send control commands to the nervous system of cockroaches $^{2}$ or rats $^{3}$ as if they were robots. Although such studies can inform us about information processing and encoding in the brains of living animals ${ }^{4}$, they do pose ethical questions and can be technically problematic since access to the brain is limited by barriers such as the skin and skull, and data interpretation is confounded by many factors including the sheer number of neurons present in the brain of even the neurophysiologically simplest animal. Moreover, whole animal approaches capable of recording the activity of individual neurons or their small populations are limited by the invasive, and hence destructive, nature of such techniques. For these reasons, neurons cultured under laboratory conditions on a planar array of non-invasive electrodes provide a far more attractive platform for probing the operation of biological neuronal networks.

This area of research is vital for a number of reasons. Firstly, understanding neural behaviour is important in establishing better bi-directional interactions between the brain and external devices. Secondly, in dealing with numerous neurological disorders, establishing an improved understanding of the fundamental basis for the manifestation of neuronal activity as meaningful behaviour is critical. A robot body can potentially move around a defined area and the effects within a biological brain, which is controlling the body, can be witnessed. This opens up the possibility of gaining a fundamental appreciation and understanding of the cellular corelates of memory and considered actions based on learning and habit.

Recent research has focussed on culturing networks of some tens of thousands of brain cells grown in vitro ${ }^{5}$. These cultures are created by dissociating neurons obtained from foetal rodent cortical tissue using enzymes and then culturing them in a specialised chamber by providing suitable environmental conditions and nutrients. An array of electrodes is embedded in the base of the chamber (a multi-electrode array (MEA)) providing an electrical interface to the neuronal culture $^{6-9}$. The neurons in such cultures begin to spontaneously extrude cellular processes, and within an hour of placement, even without external stimulation, they begin to re-connect with other nearby neurons and commence both chemical and electrical communication. This propensity to spontaneously connect and communicate demonstrates an innate tendency to network. Studies of neural cultures demonstrate distinct periods of development defined by changes in activity which appear to stabilise after 30 days and lasts for at least

Received 22 September 2009 
2-3 months ${ }^{10,11}$. The neuronal culture forms a monolayer upon the MEA on the base of the chamber, making them particularly amenable to optical microscopy and accessible to both physical and chemical manipulation?

The objective of the present project is to investigate the use of cultured neurons for the control of mobile robots. However, to produce useful processing, it is postulated that disembodied biological networks must develop in the presence of meaningful input/output relationships as part of closed-loop sensory- interaction with the environment. This is supported by animal and human studies which show that development in a sensory deprived environment results in poor or dysfunctional neural circuitry ${ }^{13,14}$. The overall closed-loop hybrid system involving a primary cortical culture on an MEA and a mobile robot ensures a sufficiently rich and consistent environment for the culture, and hence constitutes an interesting and novel approach to examine the computational capabilities of biological networks ${ }^{15}$. Typically, in vitro neuronal cultures consist of thousands of neurons generating highly variable and multi-dimensional signals. To extract components and features representative of the network's overall state from such data, appropriate pre-processing and dimensionality reduction techniques must be applied.

Several schemes reported in the literature have thus far been constructed to investigate the control capacity of hybrid systems. Notably, Shkolnik ${ }^{16}$ created an interesting control scheme for a simulated robot. Two channels of an MEA were selected and an electrical stimulus consisting of a $+/-600 \mathrm{mV}, 400 \mu \mathrm{s}$ biphasic pulse was delivered at varying inter-stimulus intervals. The concept of information coding was formed by testing the effect of electricallyinduced neuronal excitation with a given time delay termed the inter-probe interval (IPI) between two stimulus probes. This technique gives rise to a characteristic response curve which forms the basis for deciding the robot's direction of movement using basic commands (forward, backward, left, and right).

DeMarse $^{17}$, et al. have used a simulated rat which moved inside a four-wall environment including barrier objects. In other experiments ${ }^{16}$, physical robots such as 'Koala' and 'Khepera' were used in an experiment wherein one of the robots (the Koala) was able to maintain a constant distance from the Khepera robot, which was moving under random control. It was reported that the Koala robot managed to successfully approach the Khepera and maintain a fixed distance from it. It is important to stress here that spontaneous activity of the culture was sent to a computer which then made a binary decision as to what action the Koala should take. Importantly, the culture itself was not directly controlling the Koala through a feedback loop and no learning effect was reportedly exploited. In contrast, both closed-loop control and learning are central aims in the present study.

In a well publicised experiment, DeMarse and Dockendorf ${ }^{18}$ also investigated the computational capacity of cultured networks by introducing the idea of implementing the control of a "real-life" problem, such as controlling a simulated aircraft's flight path (e.g., altitude and roll adjustments). Meanwhile, recent developments have focused on the application of learning techniques in neuronal cultures. Shahaf and Marom ${ }^{19}$ reported one of the first experiments to achieve desired discrete output computations by applying a simple form of supervised learning to disembodied neuronal cultures. Recently, Bull and Uroukov ${ }^{20}$ successfully applied a learning classifier system to manipulate culture activity towards a goal level using simple input signals. However, in both cases, the desired results were only achieved in about one-third of experiments, demonstrating the underlying complexity of neuronal networks, the influence of experimental variability, and the difficulties in achieving repeatability in these systems.

Nonetheless, it is clear that even at such an early stage, such re-embodiments (real or virtual) have a prevailing role in the study of biological learning mechanisms. The proposed physical and simulated robots provide the starting point for creating a proof-of-concept control loop around the neuronal culture and a basic platform for future, more specific, reinforcement learning experiments. As the fundamental problem is the coupling of the robot's goals to the culture's input-output mapping, the design of the robot's architecture discussed in this paper emphasises the need for flexibility and the use of machine learning techniques in the search of such coupling.

\section{CULTURE PREPARATION}

To create the cultured neural network, cortical tissue was dissected from the brains of embryonic rats and neuronal cells enzymatically dissociated before seeding onto planar MEAs. Cells were restricted to lie within the recording horizon of the electrode array by an inverse template constructed from adhesive tape placed on the MEA prior to seeding and was removed immediately after cells were settled ( $1 \mathrm{~h})$. The MEA was also filled with a conventional cell culture medium containing nutrients, growth hormones, and antibiotics of which 50 per cent was replaced twice weekly. Within $1 \mathrm{~h}$ of seeding, neurons appeared to extend connections to nearby cells and within $24 \mathrm{~h}$, a dense mat of neuronal extensions was visible across the seeded area.

This connectivity further increased over subsequent days. After 7 days, initial electrical signals were observed in the form of single action potentials which, in the disembodied culture (not connected within the closed-loop) and over the following week, transformed into dense bursts of almost simultaneous electrical activity across the entire network which continued through to maturity ( 30 days in vitro and onwards). However, such continued bursting behaviour, after this initial development phase, may subsequently be representative of an underlying pathological state resulting from impoverished sensory input and may differ from activity of a culture developing within a closed loop ${ }^{21}$.

On an average, cultures remained highly active until approximately 3 months of age. During this time, they were sealed with Potter rings ${ }^{22}$ to maintain sterility and osmolarity, and were maintained in a humidified, $37^{\circ} \mathrm{C}, 5$ per cent $\mathrm{CO}_{2}$ 
incubator. Recordings were undertaken in a non-humidified $37^{\circ} \mathrm{C}, 5$ per cent $\mathrm{CO}_{2}$ incubator for between $30 \mathrm{~min}$ and $8 \mathrm{~h}$, depending on environmental humidity and the resulting stability of the activity.

\section{EXPERIMENTAL SET UP}

The MEAs enables voltage fluctuations (relative to a reference ground electrode outside the network) to be recorded at 59 sites out of 64 in an $8 \times 8$ array (Fig. 1), allowing the detection of neuronal action potentials within a $100 \mu \mathrm{m}$ radius around an individual electrode. Using spike sorting algorithms ${ }^{12}$, it may possible (although non-trivial) to separate the firing of multiple individual neurons, or small groups of neurons, from a single electrode. Consequently, multi-electrode recordings permit a picture of the global activity of the entire neuronal network to be formed. It is also possible to electrically stimulate via any of the electrodes to induce focussed neural activity. The MEA therefore forms a functional and non-destructive bidirectional interface to the cultured neurons.

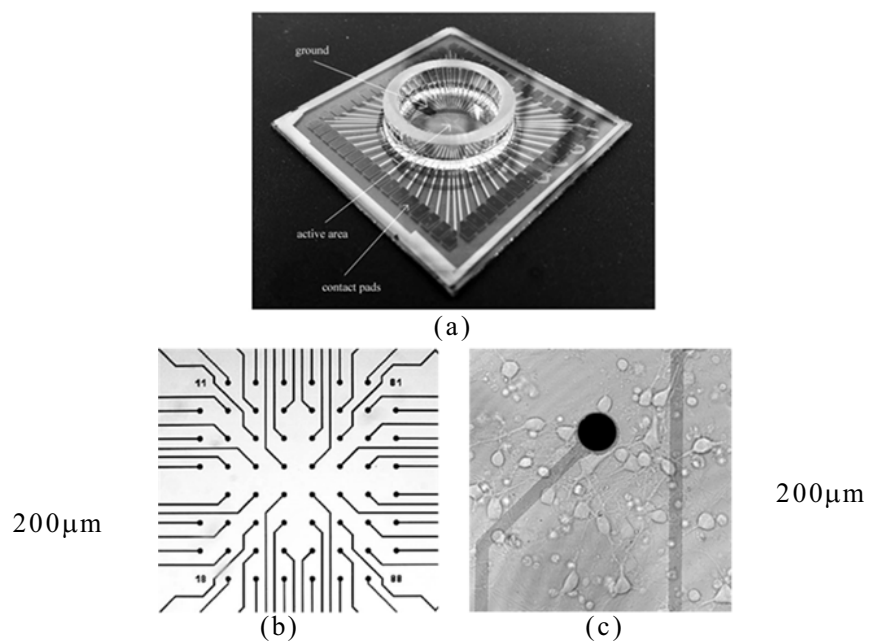

Figure 1. (a) An MC200/30iR-gr MEA (NMI, Reutlingen, Germany, UK), showing the $30 \mathrm{~mm}$ electrodes which lead to the electrode column-row arrangement (b) Electrode arrays in the centre of the MEA seen under an optical microscope (Nikon TMS, Nikon, Japan), $x 4$ magnification and (c) An MEA at $x 40$ magnification, showing neuronal cells in close proximity with visible extensions and interconnections.

The electrically-evoked responses and spontaneous activity of the culture (the neuronal network) are coupled to the robot architecture via a machine learning interface which maps the features of interest to specific actuator commands. Associating sensory data feedback from the robot with a set of appropriate stimulation protocols delivered to the culture closes the robot-culture loop. Thus, signal processing can be broken down into two discrete sections: (a) culture-to-robot, in which an output machine learning procedure processes live neuronal activity, and (b) robotto-culture, which involves an input-mapping process, from robot sensor-to-stimulus.
Our overall system has been designed as a closedloop, modular architecture. Neuronal networks exhibit spatiotemporal patterns with millisecond precision ${ }^{22}$, the processing of which necessitates a rapid response from neurophysiological recording and robot control systems. The software developed for this project runs on Linuxbased workstations communicating over the ethernet via fast server-client modules, thus providing the necessary speed and flexibility required when working with biological systems.

The study of cultured biological neurons in recent years has been greatly facilitated by commercially available planar MEA systems. These consist of a glass specimen chamber lined with an $8 \times 8$ array of electrodes, as shown in Fig. 1. A standard MEA (Fig. 1(a)) measures $49 \mathrm{~mm} \mathrm{x}$ $49 \mathrm{~mm} \times 1 \mathrm{~mm}$ and its electrodes provide a bi-directional link between the culture and the rest of the system. The associated data acquisition hardware includes a head-stage (MEA connecting interface), 60 channel amplifier (1200x gain; 10-3200 Hz bandpass filter), stimulus generator and PC data acquisition card.

Thus far, a modular closed-loop system between a (physical) mobile robotic platform and a cultured neuronal network, allowing for bi-directional communication between the culture and the robot, has been sucessfully created. It is estimated that the cultures employed in the present studies consists of approximately 100,000 neurons, the actual number depending on natural density variations in proliferation post-seeding, and aim of the experiment. The spontaneous electrochemical activity of the culture was used as input to the robot's actuators and the robot's (ultrasonic) sensor readings were (proportionally) converted into stimulation signals received by the culture, effectively closing the loop.

For the robotic framework, Miabot, a commercially available robotic platform (Merlin Robotics, UK), was selected which exhibits accurate motor encoder precision $(\sim 0.5 \mathrm{~mm})$ and has a maximum speed of approximately $3.5 \mathrm{~m} / \mathrm{s}$. Recording and stimulation hardware was controlled via open-source MEABench software ${ }^{24}$. Custom stimulator- control software was developed which interfaces with the commercially available stimulation hardware, with no need for hardware modification ${ }^{24}$.

A simulated counterpart for the real-life robot and its environment has also been developed. The simulation can interface with the culture software in exactly the same manner as the real robot system, thereby extending the modular capabilities of the system. It is expected that this simulation will be particularly helpful in long-running experiments where a real robot could face issues such as battery depletion, as well as in the deployment of various machine learning experiments explained later. It must be stressed here that the key drive for this work is for the culture to directly control the physical Miabot robot (Fig. 2). The simulation has been created as an auxiliary tool to this end and is principally useful for system set up and to ensure that the systems are running appropriately. 
The overall closed-loop system therefore consists of several modules including the real-life or simulated robot, the MEA and stimulating hardware, a directly linked workstation for conducting computationally expensive neuronal data analyses and a separate workstation running the robot control interface; a network manager routing signals directly between the culture and the robot body. The various components of the architecture communicate via TCP/IP sockets, allowing for the distribution of processing loads to multiple machines throughout the University of Reading's internal network. The modular approach to the problem is shown in more detail in Fig. 3.

The Miabot robot is wirelessly controlled via bluetooth. Communication, control are performed through custom $\mathrm{C}++$ server code and TCP/IP sockets and clients running on the acquisition PC which has direct control of the MEA recording and stimulating software. The server sends motor commands and receives sensory data via a virtual serial port over the bluetooth connection, while the client programs contain the closed-loop code which communicates with and stimulates the MEA culture.

The client code also performs text logging of all important data during an experiment run, which can then be analysed offline with the use of custom written Matlab (Mathworks, USA) tools. This modular approach to the architecture has resulted in a system with easily re-configurable components. The obtained closed-loop system can efficiently handle the information-rich data that is streamed via the recording software. A typical sampling frequency of $25 \mathrm{kHz}$ of the

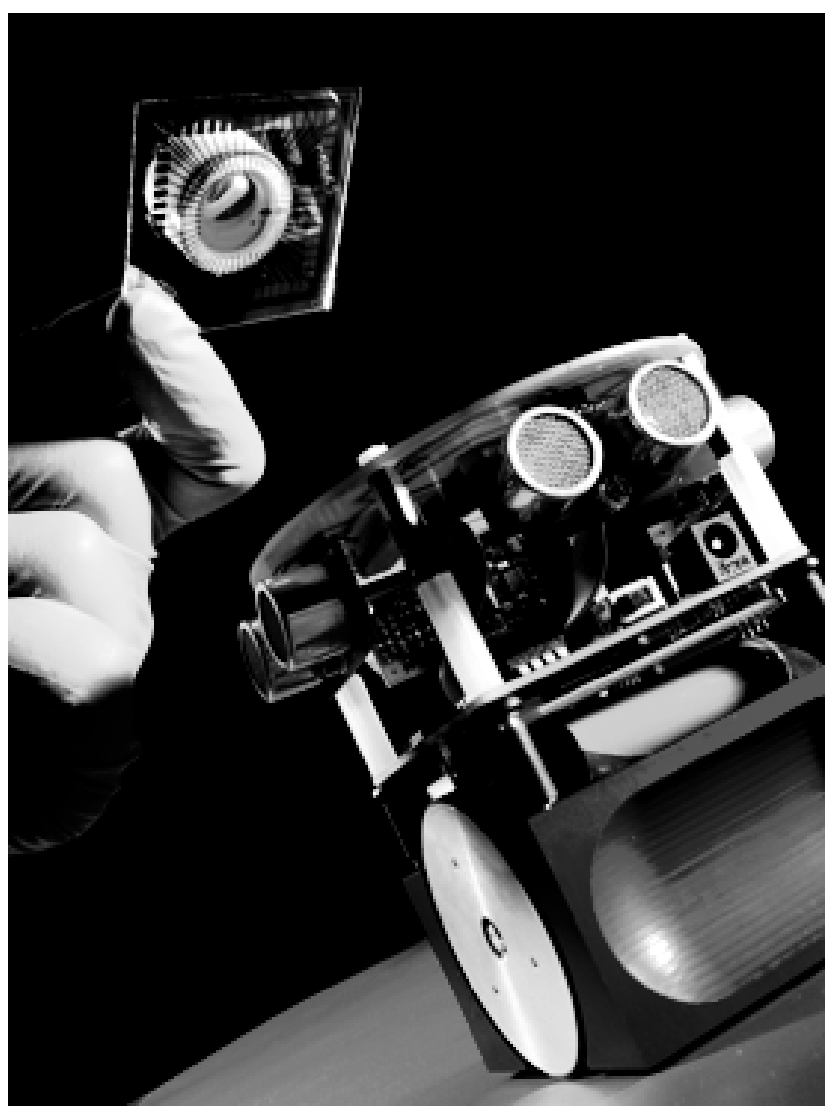

Figure 2. Miabot robot with a cultured neural network.
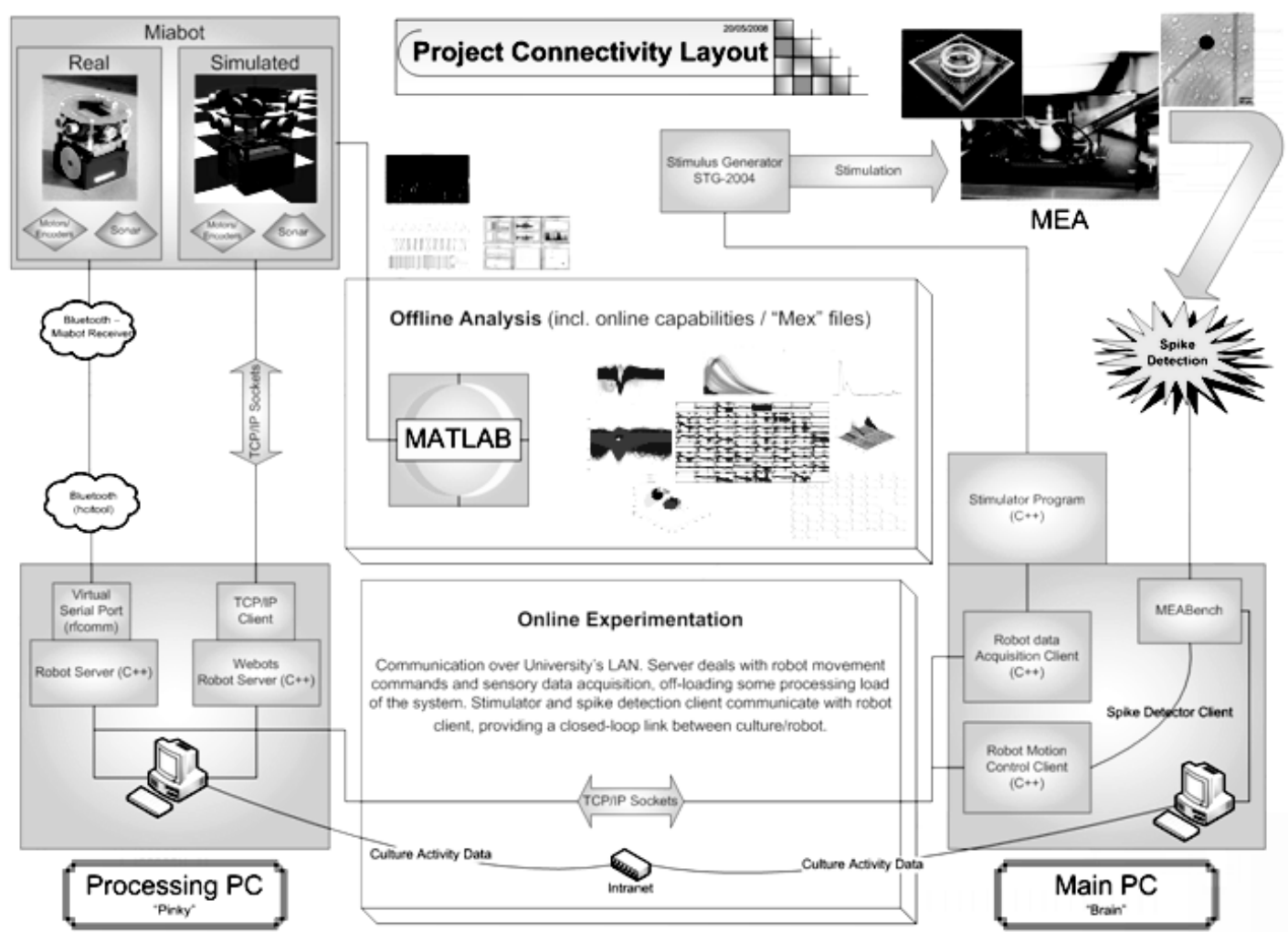

Figure 3. Modular layout of the robot/MEA system. 
culture activity demands large network, processing, and storage resources. Consequently, on-the-fly streaming of spike-detected data is the preferred method when investigating real-time closed-loop learning techniques.

Initially, all system components and operation of the entire closed loop in experiments utilising the custom stimulation software and a model cell were tested ${ }^{25}$. The model cell is a simple electronic circuit which allows for an ideal (artificial) response event within quick succession of stimulation $(<100 \mathrm{~ms})$.

The same experiment was then conducted with a live culture. To this end, an existing appropriate neuronal pathway was identified by searching for strong input/output relationships between pairs of electrodes. Suitable input/output pairs were defined as those electrode combinations in which neurons proximal to one electrode responded to stimulation of the other electrode at which the stimulus was applied (at least one action potential within $100 \mathrm{~ms}$ of stimulation) more than 60 per cent of the time and responded no more than 20 per cent of the time to stimulation on any other electrode. An input-output response map was then created by cyclic stimulation of all pre-selected electrodes individually with a positivefirst biphasic waveform $(600 \mathrm{mV} ; 100 \mu$ s each phase, repeated 16 times). By averaging over 16 attempts, this ensures that the majority of stimulation events fall outside any inherent culture bursting that may occur. In this way, a suitable input/output pair could be chosen, dependent on how the cultures had developed, to provide an initial decision making mechanism for the robot.

The robot followed a forward path within its corral confines until it reached a wall, at which point the front sonar value decreased below a threshold (set at approximately $30 \mathrm{~cm}$ ), triggering a stimulating pulse as shown in Fig. 4 . If the responding/output electrode registered activity following the input pulse then the robot turned to avoid the wall. Essentially, activity on the responding electrode was interpreted as a command for the robot to turn to avoid the wall. As a result, it was apparent that the robot also turned spontaneously whenever activity was registered on the response/output electrode. However, the most interesting and relevant result was the occurrence of the chain of events: wall detectionstimulation-response.

The model cell experiment provided a realistic representation of the maximum speed at which the closed loop could respond, this being subsequently tempered by the processing time of the culture itself when used in the loop rather than the model cell. Such a study opens up the possibility of investigating response times of different cultures under different conditions and how they might be affected by external influences such as electrical fields and pharmacological stimulants ${ }^{25}$. At any one time typically 25 different cultures were available and hence such comparative developmental studies are now being conducted.

With the sonar threshold set at approx $30 \mathrm{~cm}$ from a wall, a stimulation pulse was applied to the culture, via its sensory input, each time this threshold was breachedeffectively when the robot's position was sufficiently close to a wall. An indication of the robot's typical activity during a simple wall-detection/right turn experiment is shown in Fig. 4. The green trace indicates the front sonar value. Yellow bars indicate stimulus pulse times and blue/red bars indicate sonar timing/actuator command timing. These response events (single detected spike) may occur spontaneously or due to electric stimulation as a result of the sensor threshold being breached. These events are deemed meaningful only in the case when the delay between stimulation and response is $<100 \mathrm{~ms}$. In other words, this event is a strong indicator that the electric stimulation on one electrode caused a neural response on the recording electrode. The red vertical lines indicate the time that a rotation command is sent to the robot. These events are always coupled (the first one starts the right-turn rotation and the second simply ends the rotation). Only the second ones of each pair can be clearly seen here as the rotation initiation commands are overlaid by the yellow electrode firing bars (as a result of electrode firing which instantly

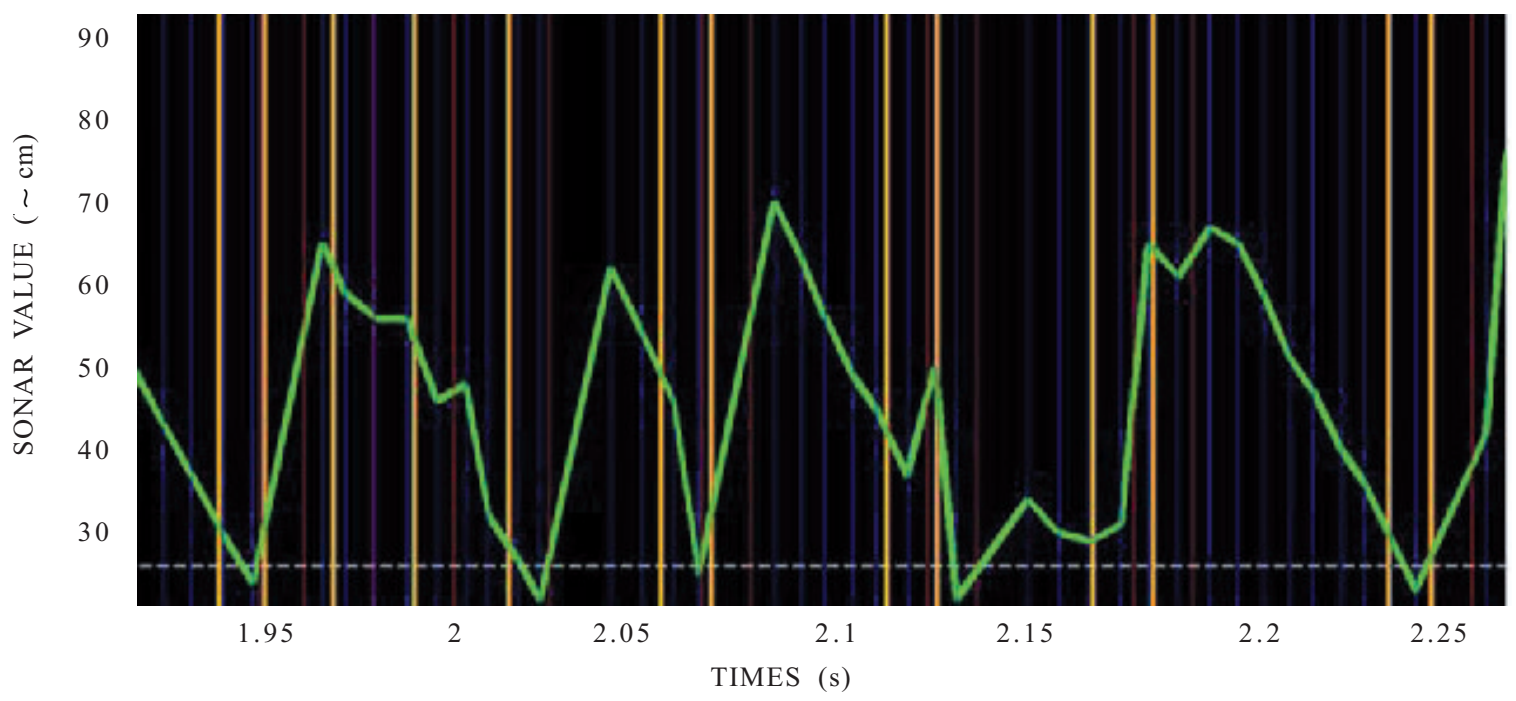

Figure 4. Analysis of the robot's activity during a simple wall-detection/right turn experiment. 
initiates a rotation command). A 'meaningful' event chain would be for example at $1.95 \mathrm{~s}$, where sonar and value drops below and threshold $(30 \mathrm{~cm})$ and a stimulation-response occurs.

Table 1 contains typical results from both a model cell and live culture test. If the live culture acted perfectly, making no mistakes, then the two columns (model cell and live culture) would be identical.

Table 1. Basic measures from a wall-avoidance experiment

\begin{tabular}{lll}
\hline Results & Model cell & Live culture \\
\hline Wall -> Stimulation event & $100 \%$ & $100 \%$ \\
Stimulation -> Response event & $100 \%$ & $67 \%$ \\
Total closed loop time & $0.075 \mathrm{~s}$ & $0.2-0.5 \mathrm{~s}$ \\
Run time & $240 \mathrm{~s}$ & $140 \mathrm{~s}$ \\
Meaningful turns & 41 & 22 \\
Spontaneous turns & 41 & 16 \\
\hline
\end{tabular}

In Table 1, the total closed-loop time refers to the time between wall detection and a response signal witnessed from the culture. Meaningful turns refers to the robot turning due to a wall detection-stimulation-response chain of events. A wall-to-stimulation event corresponds to the $30 \mathrm{~cm}$ threshold being breached on the sensor such that a stimulating pulse is transmitted to the culture. Meanwhile a stimulation-toresponse event corresponds to a motor command signal, originating in the culture, being transmitted to the wheels of the robot to cause it to change direction. It follows that for the culture, some of the stimulation-to-response events will be in considered response to a recent stimulus - termed meaningful, whereas other such events - termed spontaneous - will be either spurious or in considered response to some thought in the culture, about which there is some unawareness.

In fact, by totalling all the trials carried out thus far (>100), considerable differences (as typically indicated in Table 1) are observed between the ratio of expected and spontaneous turns between the model cell and the live culture. Under control of the model cell $95 \pm 4$ per cent (mean $\pm \mathrm{SD}$ ) meaningful turns were observed whilst the remaining spontaneous turns $(5 \pm 4 \%)$ were easily attributable to aspects of thresholding spike activity. In contrast, the live culture displayed a relatively low number of meaningful turns $(46 \pm 15 \%)$ and a large number of spontaneous turns $54 \pm 19 \%$ as a result of intrinsic spontaneous neuronal activity. Such a large number of spontaneous turns was to be expected in an uncharacterised system and current work aims to both reduce the level of ongoing spontaneous, reminiscent of epileptiform, activity present in such cultures, and to discover more appropriate input sites and stimulation patterns.

This experiment has closed the loop with the ability to apply custom stimulation protocols and has set the basis for subsequent experiments which will focus on characterising the culture responses using machine learning techniques for performing more complex robot control.

As a follow up closed loop experiment, the robot's individual (right and left separately) wheel speeds were controlled via the spike firing frequency recorded from the two chosen motor/output electrodes. The frequency is actually calculated by means of the following simple principle: A running mean of spike rate from both the output electrodes was computed from the spike detector. The detected spikes for each electrode were also separated and divided by the signal acquisition time to give an instantaneous frequency value. These frequencies were then linearly mapped (from their typical range of $0-100 \mathrm{~Hz}$ ) to a range of $0-0.2 \mathrm{~m} / \mathrm{s}$ for the individual wheel linear velocities. Meanwhile, received sonar information was used to directly control (proportionally) the stimulating frequency of the two sensory/input electrodes. The typical sonar range of $0-100 \mathrm{~cm}$ was linearly re-scaled into the range $0.2-0.4 \mathrm{~Hz}$ for electrode stimulation frequencies (600 mV voltage pulses).

The overall setup is reminiscent of a simple Braitenberg mode ${ }^{26}$. However in this case, the sensor-to-speed control is mediated by the cultured network within the overall feedback loop. For comparative purposes, the experiment was performed with both real and simulated robots with run-times of approximately $30 \mathrm{~min}$. It might be felt that such a test time is not long enough to evoke directed neural pathway changes in the culture, thereby effecting plasticity between the stimulating-recording electrodes. Although this was not a major target in carrying out this part of the experiment, it has been noted elsewhere that a high frequency burst time can induce plasticity quickly ${ }^{27,28}$. As a result, the spike timing-dependent plasticity based on the coincidence of spike and stimulus were investigated.

\section{MACHINE LEARNING}

Initially, the inherent operating characteristics of the cultured neuronal network have been taken as a starting point to enable the physical robot body to respond in an appropriate fashion. The culture then operates over a period of time within the robot body in its corral area. Experimental duration, is merely dependent to experimental design. Several experiments can therefore be completed within a day, whether on the same or differing cultures.

Learning and memory investigations are at an early stage. However we were surprised to see that during the system tests with the live culture, the robot appeared to improve its performance over time in terms of its wall avoidance ability. Currently, investigations are being carried out to know whether this promising initial observation can be repeated, robustly, and subsequently quantified. What has been witnessed could suggest that neuronal structures/ pathways that bring about a satisfactory action tend to strengthen purely through a process being habitually performed. Such plasticity has been reported elsewhere, e.g., Karniel ${ }^{29}$, et al, carried out experimentation to investigate the effects of sensory deprivation on subsequent culture development. In the present case, changes were monitored and attempts 
were made to provide a quantitative characterisation relating plasticity to experience and time. The potential number of confounding variables was considerable, as the subsequent plasticity process was (most likely) dependent on such factors as initial seeding and growth near electrodes as well as environmental transients, such as feed rate, temperature and humidity.

Following the completion of these initial phases of the infrastructure setup, a significant research contribution, it is felt, lies in the application of machine learning (ML) techniques to the hybrid system's closed-loop experiments. These techniques may be applied in various areas, such as the spike-sorting process (dimensionality reduction of spike data profiles, clustering of neuronal units), the mapping process between sensory data and culture stimulation as well as the mapping between the culture activity and motor commands, and the application of learning techniques on the controlled electrical stimulation of the culture, in an attempt to exploit the cultured networks' computational capacity.

\section{CONCLUSIONS}

At this stage it can be concluded that a closed-loop adaptive feedback system involving a (physical) mobile robotic platform and a cultured neuronal network using MEA using electrophysiological methods has been successfully realised. This enables real-time bi-directional communication between the culture and the robot. A culture being employed consists of approximately 100,000 neurons, although at any one time, only a small proportion of these neurons are actively firing.

Initial trial runs were carried out with the live cultures and comparisons made with ideal model performance. It has been observed that the culture on many occasions responds as expected, on other occasions, however, it does not, and in some cases, it provides a motor signal when it is not expected to do so.

In these circumstances, the mere concept of an ideal response is difficult to address as a biological network is involved, and it should perhaps not be seen as a negative when the culture does not adhere to or achieve such an ideal. As one still knows very little about the fundamental neuronal processes that give rise to meaningful behaviours, particularly where learning is involved, one should perhaps retain more of an open mind as to a culture's performance.

The culture preparation techniques employed are constantly being refined and have lead to stable cultures that exhibit both spontaneous and induced spiking/bursting activities which develops inline with the findings of other groups (e.g., by Chiappalone ${ }^{15}$, et al. and Potter ${ }^{22}$ and DeMarse).

A stable robotic infrastructure has also been set up, tested and is in place for future ML and culture behaviour experiments. The embodiment module can be instantiated via either a robotic hardware platform or as a software simulation. The existing, successfully tested infrastructure could be easily modified to investigate culture-mediated control of a wide array of alternative robotic devices, such as a robot head, an autonomous vehicle, robotic arms/ grippers, mobile robot swarms, and multi-legged walkers.

In terms of robotics, this study and others like it, show that a robot can have merely a biological brain to make decisions. The 100,000 neuron basis is merely due to present-day limitations - clearly this will increase. This whole area of research is therefore a rapidly expanding one as the range of sensory inputs is expanded and the number of cultured neurons encapsulated rises. The potential capabilities of such robots, including the range of tasks they can perform, therefore needs to be investigated.

\section{FUTURE RESEARCH}

There are a number of ways in which the current research programme can be taken forward. Firstly the Miabot can be extended to include additional sensory devices such as extra sonar arrays, audio input, mobile cameras and other range-finding hardware such as an onboard infra red sensor. This could provide an opportunity to investigate sensory fusion in the culture and to perform 'behavioural' experiments, possibly even attempting to demonstrate links between behaviour and culture plasticity, along the lines of Karniel ${ }^{29}$, et al. as different sensory inputs are marshalled.

Provision of a powered-floor for the robot's corral is also important, to provide the robot with relative autonomy for a longer period of time while the suggested machine learning techniques are applied and the culture's behavioural responses are monitored. For this, the Miabot must be adapted to operate on an in-house powered floor, providing the robot with an unlimited power supply. This feature, which is based on an original design for displays in museums ${ }^{30}$, is necessary since machine learning and culture behaviour tests will be carried out for many minutes and even hours at a time. It is worth pointing out however that the robotic simulation provides an alternative solution to continuous operation of the closed loop avoiding current hardware limitations.

Current hard-coded mapping between the robot goals and the culture input/output relationships can be extended using machine learning techniques to reduce, or even eliminate, the need for an a priori choice of the mapping. In particular, modern reinforcement learning techniques can be applied to various mobile robot tasks such as wall following and maze navigation, in an attempt to provide a formal framework within which the actual learning capabilities of the neuronal culture will be introduced.

To increase the effectiveness of culture training beyond the $\sim 30$ per cent success rate seen in previous work, biological experiments are currently being performed to identify physiological features which may play a role in cellular correlates of learning processes. These experiments also investigate possible methods of inducing an appropriate receptive state in the culture that may allow greater control over its processing abilities and the formation of memories ${ }^{26}$ involving specific network activity changes (switch between input and feedback states), which may allow identification of the function of given network ensembles. A further area 
of research is to identify the most suitable stage of development at which to place cultures within the closed-loop and whether a less pathological (epileptiform), and therefore more effectively manipulated, state of activity is achieved when cultures are allowed to undergo initial development in the presence of sensory input.

Also, progression of the project requires benchmarking both the machine learning techniques and the results obtained by the culture. To achieve this, it is necessary to develop a model of the cultured neural network based on experimental data about culture density and activity. This behavioural evaluation model is likely to provide insight into the workings of the neuronal network by comparing the model's and culture's performance. In particular, there is hope to gain a better understanding of the contribution of culture plasticity and learning capacity to the observed control proficiency.

\section{ACKNOWLEDGEMENTS}

This work is funded by the UK Engineering and Physical Sciences Research Council (EPSRC) under Grant No. EP/ D080134/1. The team wishes to thank the Science Museum (London), and in particular, Louis Buckley, for their housed display explicitly on this work from October 2008 onwards. The authors also wish to thank New Scientist for its general popular coverage of the authors' robot system ${ }^{32}$.

\section{REFERENCES}

1. Reger, B.; Fleming, K.; Sanguineti, V.; Simon Alford, S. \& Mussa-Ivaldi, F. Connecting brains to robots: An artificial body for studying the computational properties of neural tissues. Artificial Life, 2000, 6, 307-24.

2. Holzer, R.; Shimoyama, I. \& Miura, H. Locomotion control of a bio-robotic system via electric stimulation. In Proceedings of International Conference on Intelligent Robots and Systems, Grenoble, France, 1997.

3. Talwar, S.; Xu, S.; Hawley, E.; Weiss, S.; Moxon, K. \& Chapin, J. Rat navigation guided by remote control. Nature, 2002, 417, 37-38.

4. Chapin, J.; Moxon, K.; Markowitz, R. \& Nicolelis, M. Real-time control of a robot arm using simultaneously recorded neurons in the motor cortex. Nature Neuroscience, 1999, 2, 664-70.

5. Bakkum, D.J.; Shkolnik, A.; Ben-Ary, G.; DeMarse, T. $\&$ Potter, S. Removing some 'A' from AI: Embodied cultured networks. Lecture Notes in Comp. Sci., 2004, $130-45$.

6. Thomas, C.; Springer, P.; Loeb, G.; Berwald-Netter, Y. \& Okun, L. A miniature microelectrode array to monitor the bioelectric activity of cultured cells. Exp. Cell Res., 1972, 74, 61-66.

7. Gross, G. Simultaneous single unit recording in vitro with a photoetched laser deinsulated gold multimicroelectrode surface. IEEE Trans. Biomed. Eng., 1979, 26, 273-79.

8. Pine, J. Recording action potentials from cultured neurons with extracellular microcircuit electrodes. J. Neurosci. Meth., 1980, 2, 19-31.
9. Potter, S.; Lukina, N.; Longmuir, K. \& Wu, Y. Multisite two-photon imaging of neurons on multi-electrode arrays. SPIE Proceedings, 2001, 4262, 104-10.

10. Gross, G.; Rhoades, B. \& Kowalski, J. Dynamics of burst patterns generated by monolayer networks in culture. In Neurobionics: An Interdisciplinary Approach to Substitute Impaired Functions of the Human Nervous System, 1993, 89-121.

11. Kamioka, H.; Maeda, E.; Jimbo, Y.; Robinson, H. \& Kawana, A. Spontaneous periodic synchronized bursting during the formation of mature patterns of connections in cortical neurons. Neuroscience Letters, 1996, 206, 109-12.

12. Lewicki, M. A review of methods for spike sorting: The detection and classification of neural action potentials. Network (Bristol), 1998, 9(4), R53.

13. Saito, S.; Kobayashik, S.; Ohashio, Y.; Igarashi, M.; Komiya, Y. \& Ando, S. Decreased synaptic density in aged brains and its prevention by rearing under enriched environment as revealed by synaptophysin contents. J. Neurosci. Res., 1994, 39, 57-62.

14. Ramakers, G.J.; Corner, M.A. \& Habets, A.M. Development in the absence of spontaneous bioelectric activity results in increased stereotyped burst firing in cultures of dissociated cerebral cortex. Exp. Brain Res.,1990, 79, 157-66.

15. Chiappalone, M.; Vato, A, Berdondini, L, KoudelkaHep, M. \& Martinoia, S. Network Dynamics and Synchronous Activity in cultured Cortical Neurons. Int. J. Neural Sys., 2007, 17(2), 87-103.

16. Shkolnik, A.C. Neurally controlled simulated robot: applying cultured neurons to handle an approach/ avoidance task in real time, and a framework for studying learning in vitro, in Mathematics and Computer Science. Dept. of Computer Science, Emory University, Georgia, 2003, (Masters Thesis).

17. DeMarse, T.; Wagenaar, D.; Blau, A. \& Potter, S. The neurally controlled animal: Biological brains acting with simulated bodies. Autonomous Robots, 11, 2001, 305-10.

18. DeMarse, T.B. \& Dockendorf, K.P. Adaptive flight control with living neuronal networks on microelectrode arrays. In Proceedings of IEEE International Joint Conference on Neural Networks (IJCNN '05). 2005. pp.1549-551.

19. Shahaf, G. \& Marom, S. Learning in networks of cortical neurons. Journal of Neuroscience, 2001, 21(22), 8782788.

20. Bull, L. \& Uruokov, I. Initial results from the use of learning classifier systems to control in vitro neuronal networks. In Proceedings of the 9th Annual Conference on Genetic and Evolutionary Computation. (GECCO), 2007, ACM, London. pp. 369-76,

21. Hammond, M.; Marshall, S.; Downes, J.; Xydas, D.; Nasuto, S.; Becerra, V.; Warwick, K. \& Whalley, B.J. Robust methodology for the study of cultured neuronal networks on MEAs. In Proceedings 6th International Meeting on Substrate-Integrated Micro Electrode Arrays, 
2008. pp. 293-94.

22. Potter, S.M. \& DeMarse, T.B. A new approach to neural cell culture for long-term studies. J. Neurosci. Methods, 2001, 110, 17-24.

23. Rolston, J.D.; Wagenaar, D.A. \& Potter, S.M. Precisely timed spatiotemporal patterns of neural activity in dissociated cortical cultures. Neuroscience, 2007, 148, 294-03.

24. Wagenaar, D.A.T. \& Potter, S.M. MEABench: A toolset for multi-electrode data acquisition and on-line analysis. In Proceedings of 2nd International IEEE EMBS Conference on Neural Engineering., 2005. pp. 518-21.

25. Xydas, D.; Warwick, K, Whalley, B.; Nasuto, S.; Becerra, V.; Hammond, M.; \& Downes, J. Architecture for living neuronal cell control of a mobile robot. In Proceedings of European Robotics Symposium EUROS08, 2008, Prague. pp. 23-31.

26. Hutt, B.; Warwick, K. \& Goodhew, I. Emergent behaviour in autonomous robots, Chapter 14. In information transfer in biological systems: Design in nature series, Vol.2, edited by J. Bryant; M. Atherton \& M.Collins. WIT Press. 2005.
27. Hasselmo, M.E. Acetycholine and learning in a cortical associative memory source. Neural Comp. Arch., 1993, 5, 32-44.

28. Cozzi, L.; Chiappalone, M.; Ide, A., Novellino, A.; Martinoia, S. \& Sanguineti, V. Coding and decoding of information in a bi-directional neural interface. Neurocomputing, 2005, 65/66, 783-92.

29. 28. Novellino, A.; Cozzi, L.; Chiappalone, M.; Sanguinetti, V. \& Martinoia, S. Connecting neurons to a mobile robot: An in vitro bi-directional neural interface. Compu. Intelli. Neurosci., 2007.

30. Karniel, A.; Kositsky, M.; Fleming, K.; Chiappalone, M.; Sanguinetti, V.; Alford, T \& Mussa-Ivaldi, A. Computational analysis in vitro: Dynamics and plasticity of a neuro-robotic system. J. Neural Eng., 2005, 2, S250-S265.

31. Hutt, B. \& Warwick, K. Museum robots: Multi-robot systems for public exhibition. In Proceedings of the 35th International Symposium on Robotics, Paris. 2004. pp. 52.

32. Marks, P. Rat-brained robots take their first steps. New Scientist, 2008, 199(2669), 22-23.

\section{Contributors}

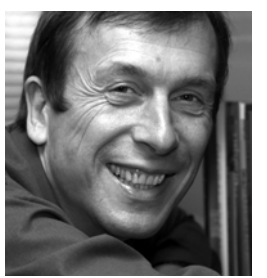

Prof K. Warwick received first degree from Aston University, followed by a $\mathrm{PhD}$ and research post at Imperial College, London. Kevin has been awarded DSc both by Imperial College and the Czech Academy of Sciences, Prague, and received Honorary Doctorates from Aston University and Coventry University in 2008. He is Professor of Cybernetics at the University of Reading, England, where he carries out research in artificial intelligence, control, robotics and cyborgs. Kevin's experiments into implant technology led to him being featured as the cover story on the US magazine, 'Wired'. Kevin's most recent research involves the invention of an intelligent deep brain stimulator to counteract the effects of Parkinson Disease tremors.

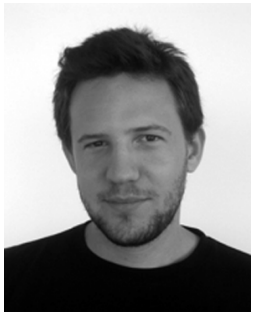

Mr D. Xydas obtained his BEng Hons in Electronic Engineering \& Cybernetics and MSc in Cybernetics in 2006, and 2007 respectively from the University of Reading, UK. As part of his degree qualifications he was involved in the longterm cross-departmental (Animat) project investigating the computational capabilities of cultured neuronal networks. At present he is a $\mathrm{PhD}$ student.

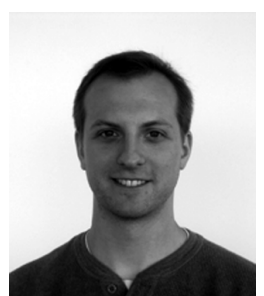

Dr S.J. Nasuto is a Reader in Cybernetics in the School of Systems Engineering at the University of Reading. Dr Nasuto's long-term research interests include investigation of the temporal information encoding and processing in the brain from the perspective of communication between many interacting components. His current research projects include use of long range synchronisation for investigation of memory processes using EEG, EEG based Brain Computer Interface, classification of single motor unit action potentials from the surface EMG and automatic reconstruction of neuronal structure from optical microscope stacks.

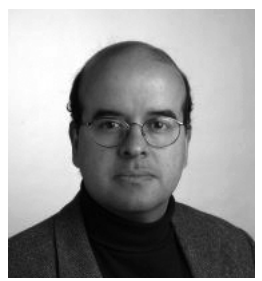

Dr V.M. Becerra obtained his first degree in Electrical Engineering in 1990 from Simon Bolivar University, Caracas Venezuela. He obtained his $\mathrm{PhD}$ for his work on the development of nonlinear optimal control methods from City University, London, in 1994. Between 1994 and 1999 he was a Research Fellow at the Control Engineering Research Centre at City University, London. He is currently a Reader in Cybernetics at the University of Reading, UK. 


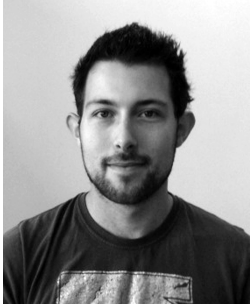

Mr M.W. Hammond obtained his Masters in Neuroscience \& Artificial Intelligence from the University of Manchester, UK, in 2005 in which his research focused on the role of theta rhythm in information flow and processing within the subiculum in vivo. He then began an RA role at the University of Reading whilst undertaking his $\mathrm{PhD}$ on a project to investigate the computational power of neuronal networks connected to a mobile robot ('Animat'). Currently his work focuses on the role of the neurotransmitter acetylcholine in learning, memory and attention within the Animat paradigm.

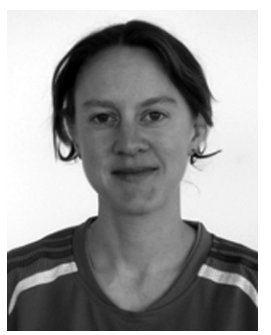

Ms J.H. Downes obtained her MSc in Computer Science from the University of North Florida, USA. Her thesis was on routing protocols for wireless sensor networks and this research was funded by the National Weather Service, USA. Subsequently she worked on signal processing for audio applications in mobile phones (Sonaptic Ltd.). Her current focus is on investigating network connectivity and measuring network connectivity changes in neuronal data.

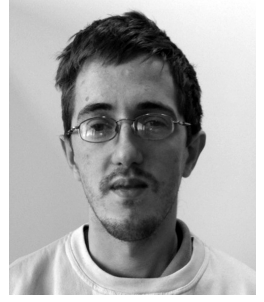

Mr S. Marshall obtained his BSc (Pharmacology) from the University of Bradford in 2005. After graduating, he started his role as a technician providing laboratory and administrative support for the researchers on the 'Animat' project. He has recently started a new role as a research technician in a project studying the anticonvulsant effects of phytocannabinoids. His academic interests cover areas involving hyper-excitability and neurodegenerative diseases and researching possible treatments for these diseases.

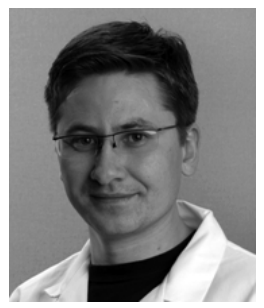

Dr B.J. Whalley is currently working as a Lecturer in Pharmacology, Reading School of Pharmacy. He has built and consolidated his own research areas that include phytocannabinoid effects upon in vitro and in vivo models of hyperexcitability disorders and in vivo appetite and feeding behaviours. He is also actively engaged in researching the computational capacity of neuronal cultures on planar electrode arrays and correlative methods between live tissue autoradiography and electrophysiology, in vitro. 